\title{
Noninvasive Fractional Flow Reserve Derived From Coronary Computed Tomography Angiography \\ - Is This Just Another New Diagnostic Test or the Long-Awaited Game Changer? -
}

\author{
Hiromasa Otake, MD, PhD; Charles A. Taylor, PhD; Hitoshi Matsuo, MD, PhD; \\ Nobuhiro Tanaka, MD, PhD; Takashi Akasaka, MD, PhD
}

\begin{abstract}
Despite the clinical importance of ischemia evaluation, obtaining fractional flow reserve (FFR) value has, heretofore, only been available during invasive cardiac catheterization. Although there is a clear linkage between morphology and physiology, a significant gap still exists. FFR derived from coronary computed tomography angiography (FFRcT) is a novel method of quantifying the hemodynamic significance of coronary artery stenoses by translating morphological information into hemodynamic data. Improved diagnostic performance of FFRct has been repeatedly demonstrated in comparison with coronary computed tomography angiography alone with an invasive FFR reference standard. More recently, the potential benefit of this technology to safely defer non-indicated catheterizations and reduce healthcare costs has been established and indicates this technology may improve the management of patients with coronary artery disease. In this review, we summarize the scientific basis of FFRct and evidence from clinical trials, provide illustrative examples of clinical applications, discuss potential limitations, and outline avenues for future research.
\end{abstract}

Key Words: Coronary computed tomography angiography; Fractional flow reserve; Ischemic heart disease

$\mathbf{F}$ or decades, the importance of ischemia detection has been repeatedly emphasized for improving clinical outcome and cost-effectiveness in the treatment of patients with coronary artery disease (CAD). ${ }^{\mathbf{1 2}}$ Unlike noninvasive functional imaging tests, fractional flow reserve (FFR) measured by a pressure wire enables ischemia detection on a per-lesion basis, giving direct information for predicting the therapeutic benefit of coronary revascularization. FFR-guided percutaneous coronary intervention (PCI) has been associated with a $28 \%$ lower rate of major adverse cardiac events compared with an angiography-guided strategy, ${ }^{1}$ with significant cost savings. ${ }^{2}$ Furthermore, FFR-guided PCI plus optimal medical therapy has been proven to decrease the need for urgent revascularization compared with optimal medical therapy alone. ${ }^{3}$ Based on these data, FFR-guided decision-making has become the gold standard for managing patients with stable CAD.

Yet, pressure-wire based FFR measurement requires an invasive procedure with intravenous or intracoronary pharmacological intervention to induce maximal hyperemia. Additionally, because invasive coronary imaging- guided PCI is a standard method in Japan, the additional time and cost for invasive FFR measurement could impair adoption of this approach. There is an unmet clinical need for an effective, noninvasive method of assessing the functional significance of coronary lesions to predict the potential benefit of revascularizing anatomically obstructive coronary stenosis.

Recently, application of computational fluid dynamics technology to coronary computed tomographic angiography (cCTA) images has enabled noninvasive computation of FFR. ${ }^{4}$ This review summarizes the scientific basis and data supporting the application of FFR CT $_{\text {to }}$ assessing CAD, its potential limitations, and directions for future research.

\section{Basic Concept of FFRct}

FFR ${ }_{\text {CT }}$ uses a mathematical model combining patientspecific imaging data with population-derived physiologic models and computational fluid dynamics to solve the governing equations of blood flow for velocity and pressure under simulated hyperemia. The equations governing

Received June 14, 2016; revised manuscript received February 8, 2017; accepted March 14, 2017; released online April 11, 2017

Division of Cardiovascular Medicine, Department of Internal Medicine, Kobe University Graduate School of Medicine, Kobe (H.O.), Japan; HeartFlow, Inc., Redwood City, CA (C.A.T.); Department of Bioengineering, Stanford University, Stanford, CA (C.A.T.), USA; Department of Cardiology, Gifu Heart Center, Gifu (H.M.); Department of Cardiology, Tokyo Medical University Hachioji Medical Center, Tokyo (N.T.); and Department of Cardiology, Wakayama Medical University, Wakayama (T.A.), Japan

Mailing address: Takashi Akasaka, MD, FACC, Department of Cardiology, Wakayama Medical University, 811-1 Kimiidera, Wakayama 641-8510, Japan. E-mail: akasat@wakayama-med.ac.jp

ISSN-1346-9843 All rights are reserved to the Japanese Circulation Society. For permissions, please e-mail: cj@j-circ.or.jp 

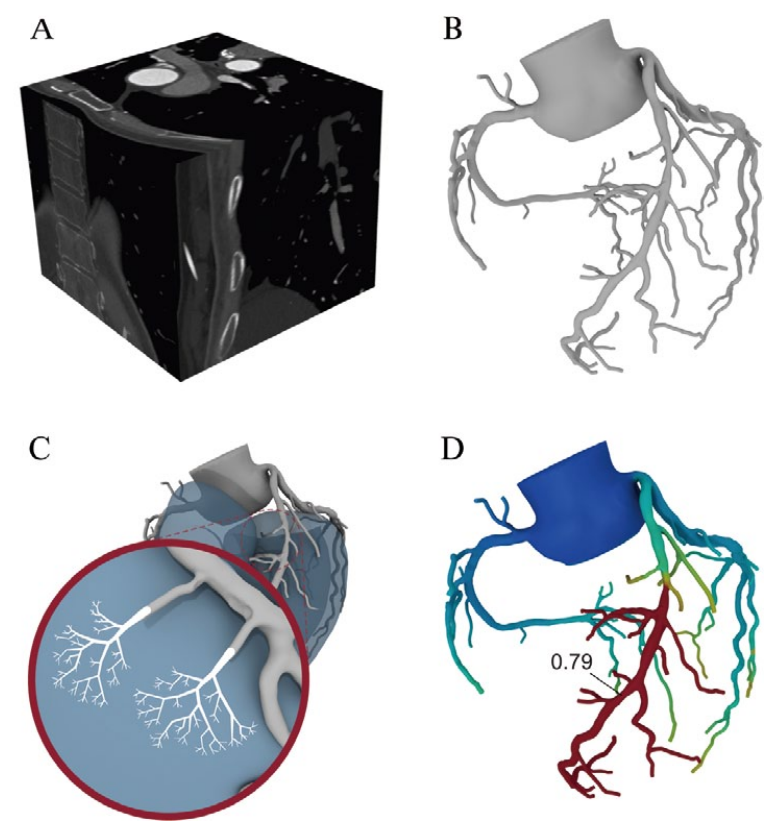

Figure 1. Methodology of fractional flow reserve (FFR) derived from coronary computed tomography angiography (FFRcT). (A) Coronary CT angiography data are acquired and assessed for image quality, (B) image-processing methods are used to segment the root of the aorta and epicardial coronary arteries down to approximately $1 \mathrm{~mm}$ diameter, $(\mathbf{C})$ the left ventricular myocardial volume is extracted from the image data and a model of the microcirculation is created based on epicardial vessel size, and (D) computational fluid dynamics methods are used to simulate blood flow under hyperemic conditions and calculate the FFR. FFRcr is displayed throughout the coronary arteries and numerical values are extracted.

blood flow in arteries, the Navier-Stokes equations are conceptually simple - a statement of conservation of mass and a generalization of Newton's second law, $F=m a$, to a fluid - but mathematically complex, a system of nonlinear partial differential equations. Recently, computer methods to solve the Navier-Stokes equations and simulate blood flow in patient-specific models of arteries have been developed and applied to the coronary arteries.,5

Figure 1 depicts the steps involved in the HeartFlow FFRCT analysis. Upon acquisition of cCTA data and assessment of image quality, a 3D model of the proximal aorta and coronary arteries is extracted using image segmentation algorithms. Next, the left ventricular myocardial volume is extracted for assessment of total coronary blood flow demand. A physiologic model representing aortic pressure and microcirculatory resistance is defined first under baseline (resting) and then under maximal hyperemic conditions. Blood flow and pressure are solved in the patient-specific model under conditions of simulated maximal hyperemia and FFR $\mathrm{CT}$ is extracted from the pressure field as the ratio of mean coronary to mean aortic pressure. The methodology and scientific basis of this technology are explained in more detail in the following.

\section{Creating Patient-Specific Anatomic Models From cCTA Data}

Pressure losses along the coronary artery are determined by the vessel's anatomy and the blood flow from the ostia to the distal measurement point. Minimum lumen area, lesion length, serial lesions and diffuse disease affect coronary flow and pressure. Additionally, the perfusion territory downstream of a coronary lesion affects the flow through that lesion and thus the pressure loss. As a result, creation of patient-specific anatomic models of the coro-

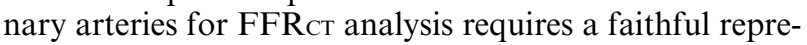
sentation of lumen dimensions for the primary coronary arteries as well as the branches that define the perfusion territory downstream of a stenosis.

Image-processing methods are used to extract patientspecific geometric models of the coronary arteries and left ventricle from cCTA data. Because FFR CT $_{\text {analysis pre- }}$ dicts invasive FFR, this necessitates minimizing differences in vessel caliber between cCTA and cardiac catheterization through the use of sublingual nitrates, optimizing scanning protocols to attain adequate spatial and temporal resolution, and using robust image segmentation methods to resolve lumen dimensions even in the presence of calcium, motion and other image artifacts.

Vessel caliber influences both measured FFR and FFRct, so it is important to ensure that the vessel size at the time of cCTA matches that which would be observed were invasive FFR measurement performed. Sublingual nitrates administered just prior to the cCTA scan are needed to ensure this matching, as invasive FFR is measured after intracoronary nitroglycerine administration. Nitrates can increase the diameter of coronary arteries by $\geq 15 \%$ for large epicardial arteries and by $30-40 \%$ for smaller vessels. ${ }^{6}$ Such differences in diameter can have a marked effect on predicted pressure loss and FFR CT values along the vessel. Society of Cardiac CT (SCCT) guidelines recommend nitrates, for patients who are not contraindicated, at a preferred dose of $800 \mu \mathrm{g}$ of sublingual nitroglycerin a few minutes before the initiation of the scan protocol. ${ }^{7}$ Although tablets can be used, provided care is taken to ensure the submucosal surfaces are moist and the tablets dissolve, the metered spray provides more consistent vasodilation.

FFRCT analysis uses standard cCTA image data, ${ }^{7}$ but adherence to established guidelines, especially related to the use of $\beta$-blockers for heart rate control, is needed to obtain good quality image data for $3 \mathrm{D}$ quantitative analysis. Ideally, heart rates should be $<60$ beats/min. Furthermore, reconstruction of image data should limit the field of view to the heart to attain in-plane resolution better than $0.5 \times 0.5 \mathrm{~mm}^{2}$ and slice thickness $<1 \mathrm{~mm}^{7}$

Robust and accurate image-processing methods are needed to segment the coronary arteries and create a quantitative anatomic model. It has been argued that, because the resistance of a coronary artery segment can be approximated as inversely related to minimum lumen diameter (MLD) to the 4th power, limits in spatial resolution of cCTA could introduce large errors in stenosis diameter and larger errors in stenosis resistance, pressure drop and calculated FFR ${ }_{\mathrm{CT}}{ }^{\mathbf{8}}$ In fact, the pressure gradient is inversely related to the minimum lumen area to the 2 nd power and because cCTA is a cross-sectional imaging technique based on resolving area, this decreases the sensitivity to minimum lumen dimensions. Furthermore, the pressure gradient is also directly related to flow to the 2 nd power, which decreases with reductions in minimum lumen area. Finally, the effect of image resolution on minimum lumen area estimation is lessened by the fact that cCTA data are gray scale, not binary (black and white) images. As a result, 
subpixel (or subvoxel) resolution can be attained with computer-automated image segmentation methods (i.e., the accuracy of geometry estimation is not limited to the spatial resolution of the data). Recently, Otake and colleagues used optical coherence tomographic (OCT) data co-registered to cCTA images to demonstrate an accuracy of MLD of CT at approximately $0.26 \pm 0.19 \mathrm{~mm}$, much better than the typical image resolution. ${ }^{9}$

Although the spatial resolution of standard cCTA data is sufficient for constructing a high-fidelity anatomic model for FFR $\mathrm{FT}$ analysis, poor image quality can preclude interpretation of cCTA data and impair the image segmentation necessary for FFR ${ }_{C T}$ modeling. ${ }^{10}$ For FFRCT, image quality is determined using a vessel scoring approach to assess whether a quantitative model of that artery can be extracted from the cCTA data. The score depends on the length and number of regions where the vessel lumen boundary is determined to be uninterpretable because of imaging artifacts, including excessive motion, mis-registration, low contrast opacification or excessive, circumferential calcium. In the recent NXT and PLATFORM clinical trials, FFRст could be computed in approximately $87 \%$ of patients. Reasons for rejection related to the presence of artifacts that precluded quantitative interpretation of the lumen, including extensive calcification, poor opacification, excessive motion or severe mis-registration.

\section{Physiologic Principles Underlying FFRct}

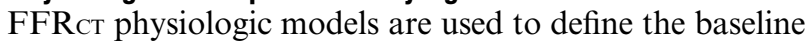
and hyperemic boundary conditions applied to the patientspecific anatomic model derived from cCTA for subsequent calculation of coronary blood flow and pressure using computational fluid dynamics methods. These physiologic models are based on 3 core scientific principles: the scaling law relating coronary flow to myocardial mass, the principle of flow regulation of vessel size, and the predictable reduction of microvascular resistance with maximal hyperemia. ${ }^{\mathbf{1 1 , 1 2}}$

The first scientific principle used in the FFRct physiologic model is that baseline coronary flow is proportional to left ventricular myocardial mass following an "allometric" scaling law, $\mathrm{Q}_{\mathrm{cor}} \propto \mathrm{M}_{\mathrm{myo}}{ }^{3 / 4}$. This principle is used with the patient's blood pressure to compute total baseline coronary artery resistance. In a porcine model, Choy et al investigated scaling of myocardial flow and mass using microspheres to quantify total coronary flow and reported an exponent of $0.74 \pm 0.04$ with an $\mathrm{r}^{2}$ value of 0.97 .13

The assumption that baseline coronary flow is proportional to myocardial mass is supported by perfusion imaging data. Absolute myocardial perfusion measurements performed using PET or MRMPI express mean flow per unit of myocardial mass (e.g., in units of $\mathrm{mL} / \mathrm{min} / \mathrm{g}$ ). Resting perfusion is fairly constant and ranges from roughly $0.82 \pm 0.06$ to $0.97 \pm 0.10 \mathrm{~mL} / \mathrm{min} / \mathrm{g}$ in different patient populations. ${ }^{\mathbf{1 4}}$

The second scientific principle in the FFR F $_{\text {physiologic }}$ model is the continuous adaptation of vessel caliber to flow, and hence that microvascular resistance can be estimated from the size of the feeding vessel. ${ }^{\mathbf{1 5}, 16}$ Such adaptive mechanisms are preserved with progression of atherosclerotic disease through compensatory remodeling of the arteries until the disease becomes so severe as to exceed the capacity for remodeling and luminal encroachment ensues. ${ }^{17}$ This form-function relationship between flow and vessel size enables determination of baseline flow and resis- tance in an epicardial coronary artery imaged with cCTA based on the size of the artery. In practice, this necessitates construction of a model of the main and branch vessels and determination of the nominal size of each vessel. The relative resistance of the circulation downstream of each epicardial coronary artery, together with the patient's total coronary arterial resistance, is sufficient to derive absolute baseline coronary artery resistance of the circulation downstream of each epicardial coronary artery. The governing equations of blood flow can then be solved for coronary velocity and pressure under baseline conditions.

The third scientific principle of FFR $\mathrm{CT}_{\mathrm{T}}$ is that the microcirculation reacts predictably at maximal hyperemia and this is supported by the data of Wilson et al demonstrating the effect of vasodilators on total coronary resistance index (defined as the hyperemic resistance divided by the resting resistance). ${ }^{18}$ The effects of varying doses of intracoronary and intravenous adenosine on total coronary resistance index were evaluated in 31 patients with atypical chest pain, normal or mildly stenotic coronary arteries, normal left ventricular function and normal coronary flow reserve as assessed using papaverine. These data from Wilson et al were used to model the maximum reduction in resistance to flow of the microvasculature downstream of each epicardial coronary artery. The governing equations of blood flow can then be solved for velocity and pressure under maximum hyperemic conditions. Note, however, that an assumption of maximum reduction in microcirculatory resistance is not equivalent to an assumption of normal coronary flow reserve because diffuse and focal atherosclerosis will diminish the coronary flow reserve despite maximal microcirculatory vasodilation.

The applicability of FFRст technology in patients with microvascular disease and reduced coronary flow reserve despite "normal" epicardial coronary arteries has been questioned. In this hypothetical case, the assumption of a normal microvascular response to adenosine could lead to a greater reduction in resistance from baseline to hyperemic states, higher flow through the model than occurs in vivo, a greater pressure drop, and therefore a potential for

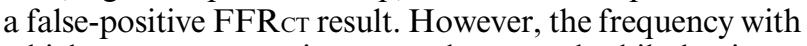
which coronary arteries are truly normal while having a diminished microvascular response to adenosine is unclear because coronary arteries are not necessarily normal when there is no angiographic evidence of disease. ${ }^{19,20}$ Because arteries adapt in response to flow, it would be expected that microvascular disease would create a feedback loop whereby increased microvascular resistance would decrease flow through the feeding vessel, which would in turn diminish in caliber. In some cases, these reductions in vessel caliber may lead to vascular rarefaction, thereby reducing the number of branches emanating from the main epicardial vessels. In addition, vessels may have diffuse CAD, which may not inhibit resting flow but could be flow-limiting under stress conditions. For example, De Bruyne et al investigated FFR in vessels free of any visible focal narrowing in patients with remote significant stenoses and found that approximately $18 \%$ of these vessels had an FFR $\leq 0.80 .^{20}$

\section{Computational Fluid Dynamics Methods Underlying FFRct}

FFR ${ }_{\text {CT }}$ uses computational fluid dynamics methods to solve the 3D governing equations of blood flow in patientspecific models of the coronary arteries subject to boundary conditions established using the physiologic principles 


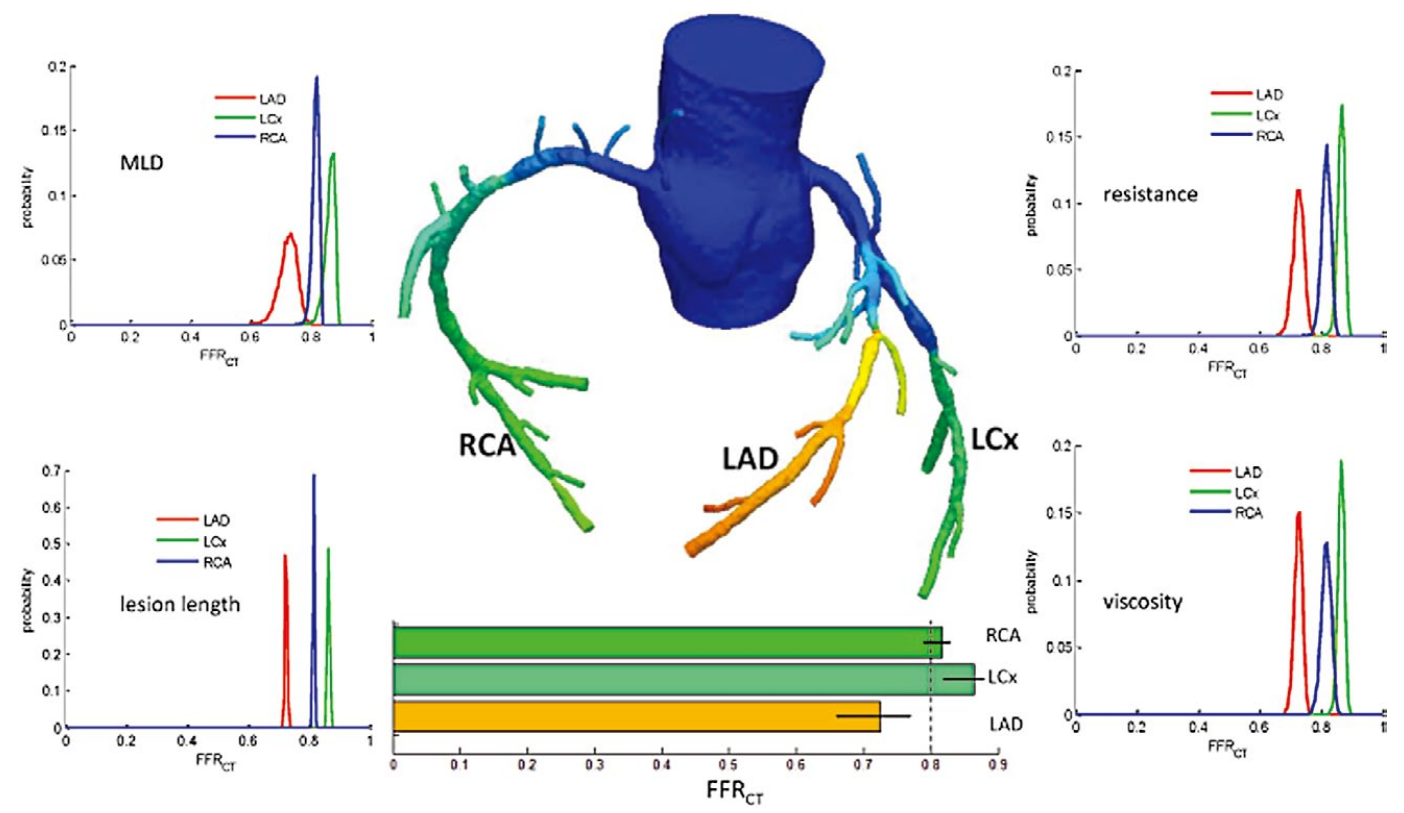

Figure 2. FFRCT map computed at the mean value of all the parameters shown together with the confidence intervals for the LAD, LCX and RCA. The figure also shows the probability distribution of FFRCT caused by uncertainty in MLD, lesion length, resistance and viscosity in the LAD, LCx and RCA. Lesion length has the smallest magnitude of uncertainty in FFRCT whereas MLD has the highest. The effect of uncertainty in boundary resistance is slightly higher than that because of uncertainty in blood viscosity. (Reproduced with permission from Sankaran S, et al. ${ }^{21}$ ) LAD, left anterior descending coronary artery; LCx, left circumflex artery; MLD, minimum lumen diameter; RCA, right coronary artery. Other abbreviation as in Figure 1.

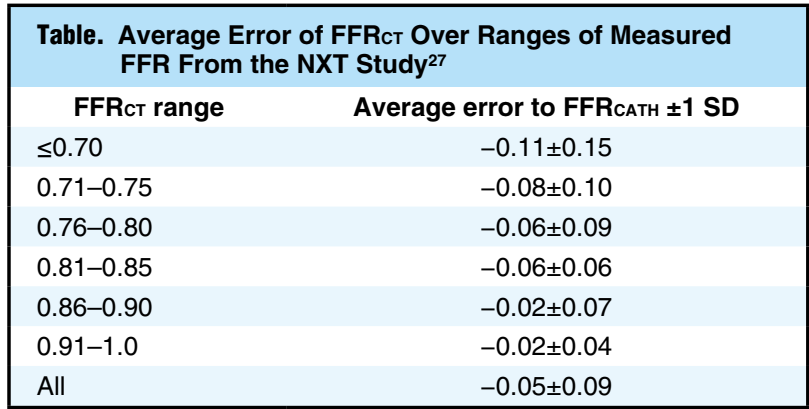

FFRct, fractional flow reserve derived from coronary computed tomography angiography; SD, standard deviation.

described before. Special-purpose computational fluid dynamics methods have evolved over the past 2 decades for use in modeling blood flow in arteries. ${ }^{5}$

\section{Assessing the Effect of Model Uncertainty on FFRcr}

Sankaran et al assessed the effect of model uncertainty on FFR ст predicted values (Figure 2). ${ }^{21}$ Uncertainty in MLD

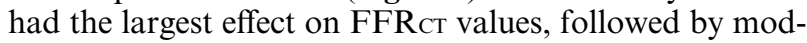
eled boundary resistance, viscosity and lesion length. Near the diagnostic cutoff $\left(\mathrm{FFR} \mathrm{CT}_{\mathrm{C}}=0.8\right)$, the uncertainty created by the latter 3 variables was lower than measurement uncertainty, whereas the uncertainty related to MLD was only slightly higher than measurement uncertainty. Uncertainties were not additive but only slightly higher than the highest single parameter uncertainty.

In summary, based on theoretical analysis and empirical data, FFRcT accuracy and precision are most affected by the fidelity of the geometric model extracted from the cCTA data. Patient preparation protocols to ensure the geometry of the patient at the time of cCTA matches that at the time of FFR measurement (i.e., a good nitrate protocol), as well as heart rate control to improve cCTA image quality, are necessary for obtaining good clinical results.

\section{Clinical Trials of FFRct}

\section{Diagnostic Performance Trials}

To date, 3 prospective, multicenter trials have been conducted to evaluate the diagnostic performance of FFRст. The diagnostic performance of FFR $\mathrm{F}_{\mathrm{C}}$ was first evaluated in the DISCOVER-FLOW trial by comparing FFRCT with cCTA stenosis in 159 vessels in 103 patients using invasive FFR as a reference standard. ${ }^{22}$ Per-vessel accuracy, sensitivity, specificity, positive predictive value (PPV), and negative predictive value (NPV) for lesions causing ischemia was $84 \%, 88 \%, 82 \%, 74 \%$, and $92 \%$, respectively for FFRct, whereas those of CTA stenosis were 59\%, 91\%, $40 \%, 47 \%$, and $89 \%$, respectively. As a result, a dramatic improvement in diagnostic accuracy was provided by FFRст ( $84 \%$ vs. $59 \%)$, with improved specificity and PPV without significant changes in sensitivity and NPV.

These findings were confirmed by the recent NXT multicenter trial including 254 suspected CAD patients from 10 sites. This trial used an updated version of FFRст with refined image-processing methods and physiological modeling improved by applying information derived from study patients in earlier trials. The NXT trial found higher 


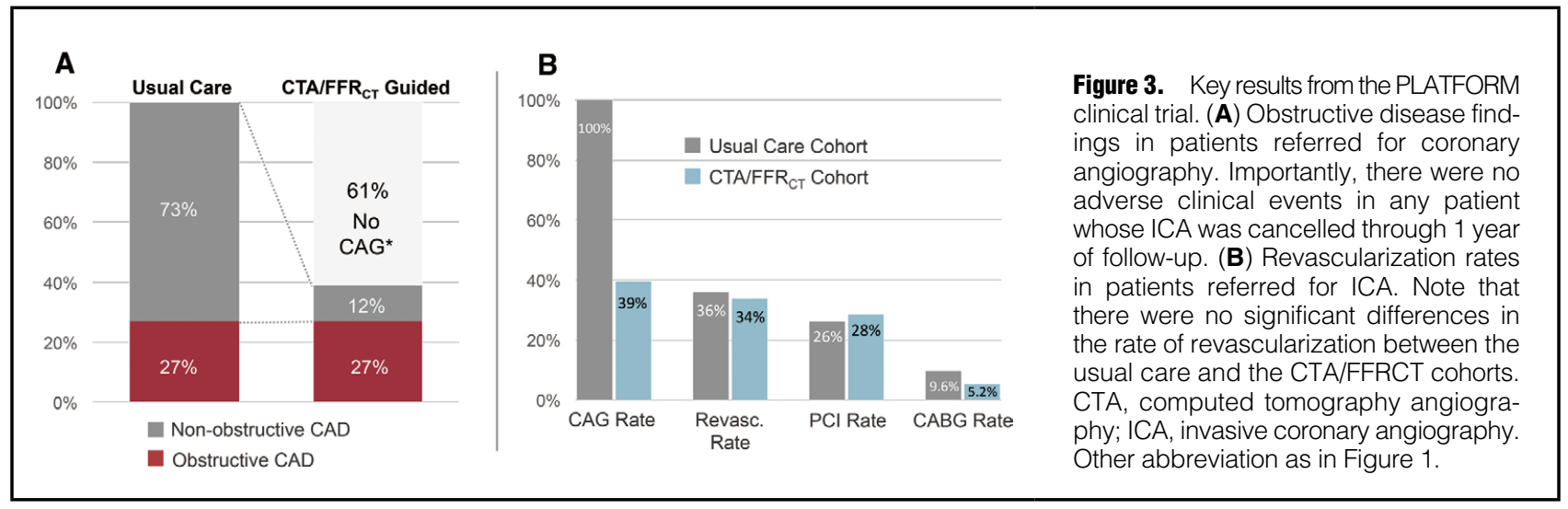

diagnostic performance of FFR $\mathrm{CT}$ compared with cCTA, with accuracy ( $81 \%$ vs. $53 \%)$, sensitivity ( $86 \%$ vs. $94 \%$ ), specificity (79\% vs. $34 \%$ ), PPV (65\% vs. $40 \%)$, and NPV $(93 \%$ vs. $92 \%)$ on a per-patient basis, respectively. ${ }^{23,24}$ When invasive FFR was used as the gold standard, data from the NXT study revealed only a $2 \%$ chance of FFR

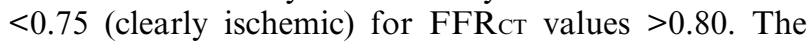
Table shows data on the error against measured FFR over ranges of FFRCT values. In general, FFRCT was biased lower than invasive FFR to avoid false-negative cases.

The NXT trial was the first trial of FFRct with a Japanese arm of 57 patients. ${ }^{25} \mathrm{~A}$ post-hoc analysis of these patients demonstrated marked improvement in the diagnostic accuracy of FFR CT compared with cCTA alone (74\% vs. $47 \%$, respectively, $\mathrm{P}<0.01)$, which was primarily driven by more than 2-fold higher specificity (FFRcт: 63\%, cCTA: 27\%, $\mathrm{P}=0.01$ ). Despite such improvement, per-patient diagnostic accuracy of the Japanese subset appeared slightly lower than that of the overall study ( $74 \%$ vs. $81 \%$, respectively), possibly because of the smaller sample size of the Japanese cohort. Another possible explanation for the difference is that the Japanese subset had a higher prevalence of patients with highly calcified lesions. In the Japanese arm, $45 \%$ of the patients had an Agatston score $>400$ compared with $26 \%$ overall. In another recent substudy of the NXT trial, however, Norgaard et al reported similar diagnostic benefit of adding FFR CT $_{\text {to }}$ CTA in patients in the highest quartile of Agatston score (416-3,599) compared with lower quartiles. ${ }^{26}$ Even in the Japanese subset, the diagnostic accuracy of patients with Agatston score $<1,000$ was similar to that in the overall NXT trial $(83 \%$ vs. $81 \%$,

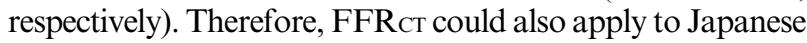
patients with CAD if the image quality is acceptable.

\section{Reproducibility of FFRct}

The reproducibility of FFRCT and invasive FFR was studied in a subset of NXT in 28 patients (58 vessels) in which invasive FFR was measured twice with pressure-wire removal and cessation of intravenous adenosine infusion during each measurement. FFRст measurement was performed using the same cCTA data set at 2 separate time points by 2 independent analysts in a blinded manner. The coefficient of variation of FFR $\mathrm{FT}_{\mathrm{T}}$ was $3.4 \%$ (95\% confidence interval: 1.44 .6 ), suggesting an acceptable performance as a biological test, while that of invasive FFR was $2.7 \%$ (95\% confidence interval: $1.8-3.3)$. This reproducibility of FFRcT remained unchanged in the subgroup of vessels with FFR values between 0.70 and $0.90 .^{27}$

\section{Importance of Image Acquisition Protocols and cCTA Image Quality}

Compliance with current guidelines of the SCCT regarding cCTA imaging protocols is important for the diagnostic performance of FFRст. Especially pertaining to the use of pre-cCTA nitrates and $\beta$-blockers, compliance with SCCT guidelines was associated with improved diagnostic performance of FFRст. In the DeFACTO trial, administration of a $\beta$-blocker and nitroglycerin increased FFR $\mathrm{FT}_{\text {specificity }}$ (66.0\% vs. $51.0 \%, \mathrm{P}=0.03$ and $75.0 \%$ vs. $54.0 \%, \mathrm{P}=0.013$ ), with a lower systematic bias observed after $\beta$-blocker administration $(-0.084$ vs. $-0.048 ; \mathrm{P}=0.008)$. The per-vessel specificity improved from $54 \%$ for patients who did not receive nitrates or those that received sublingual nitrates $>30$ min before the acquisition of cCTA data to $75 \%$ for patients administered sublingual nitrates $\leq 30 \mathrm{~min}$ before cCTA acquisition. ${ }^{11}$ In NXT, patient preparation protocols were refined from DeFACTO, resulting in $78 \%$ of patients receiving $\beta$-blockers and $99.6 \%$ of patients receiving sublingual nitrates prior to cCTA acquisition. Prior to FFRCT analysis, the image quality was assessed to clarify if the cCTA had acceptable quality for quantification of the luminal boundary. In DeFACTO and NXT, 11\% and $13 \%$ of studies, respectively, were rejected because of low image quality. ${ }^{\mathbf{1 1 , 2 4}}$

\section{Effect of FFRct on Diagnosis and Treatment Strategy, Costs, and Quality of Life}

The PLATFORM trial was a 584-patient, prospective, controlled utility trial evaluating patients with an intermediate likelihood of CAD. Those patients were divided into 2 strata based on whether an invasive or noninvasive diagnostic test was planned. In this study, in patients with planned invasive coronary angiography (CAG), a cCTA/

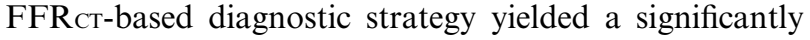
lower incidence of CAG without obstructive disease with similar mean cumulative radiation exposure (9.9 vs. $9.4 \mathrm{mSv}, \mathrm{P}=0.20$ ) as compared with a CAG-based strategy. Although all the patients were originally planned for $\mathrm{CAG}$, $61 \%$ of CAG was cancelled after receiving cCTA/FFRCT results and the rate of cases with no obstructive CAD was reduced by over $80 \%$ (from $73 \%$ to $12 \%$ ) (Figure 3A). Despite the substantial reduction in $\mathrm{CAG}$, the rate of revascularization was unchanged between the planned invasive CAG and cCTA/FFRct cohorts (Figure 3B). At 1 

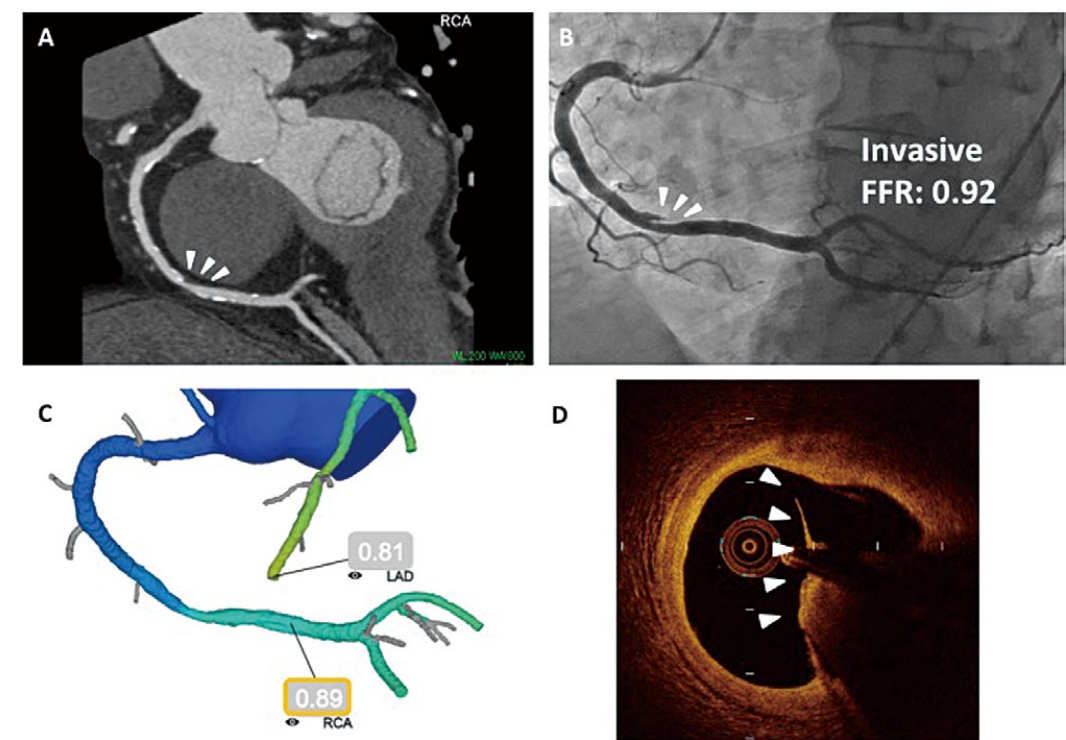

D

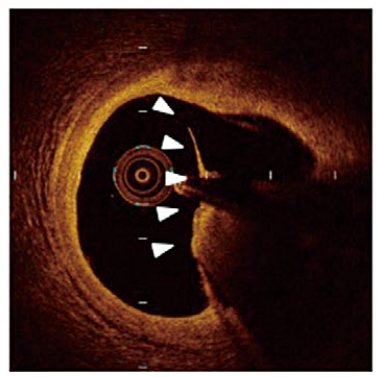

Figure 4. Case 1. (A) CTA image of RCA, (B) angiography of RCA, (C) FFRCT and (D) OCT image: white arrowhead indicates intracoronary flap. OCT, optical coherence tomography. Other abbreviations as in Figures 1-3.

year, there were no adverse events in any patient in which catheterization was cancelled on the basis of the FFRCT results. ${ }^{28}$ As a secondary analysis, among those with intended noninvasive testing there was no statistical difference in the rates of cases of no obstructive CAD between CTA/FFRCT and usual care (13\% vs. 6\%; respectively, $\mathrm{P}=0.95){ }^{29}$

Currently, more than 2 separate tests are needed in many patients to evaluate the anatomic and physiologic information..$^{30}$ In a recent simulation analyses, Hlatky et al suggested the possibility of meaningful cost savings and clinical benefits of FFRc to guide clinical decision-making under the United States healthcare system. ${ }^{31}$ They showed that use of FFRст to select patients for invasive CAG and PCI may result in 30\% lower costs and 12\% fewer events at 1 year compared with the most common strategy of CAG and visually guided PCI. Such benefits from FFRct were projected to be similar in Japan. Kimura et al reported that

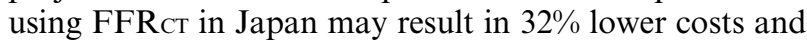
$19 \%$ fewer cardiac events at 1 year compared with a CAGbased strategy, based on data from the NXT trial with Japanese procedure and device cost information. ${ }^{32}$ These results were confirmed by a subanalysis of the PLATFORM trial, which has secondary endpoints related to costs, resource utilization, and quality of life. In patients with planned $\mathrm{CAG}$, mean costs (diagnostic tests, invasive procedures, hospitalizations, and medications during 90-day follow-up) were significantly lower among the FFRCT patients than among the usual-care patients (US\$7,343 vs. $\$ 10,734 \mathrm{P}<0.0001$ ), with $32 \%$ cost reduction by FFRст. In the sensitivity analysis, when the cost of FFRCT was set to 7-fold that of cCTA, the FFRCT group still had lower costs than the usual-care group in the invasive testing stratum (US\$8,619 vs. $\$ 10,734, \mathrm{P}<0.0001$ ). At 1 year, overall costs were reduced by more than $\$ 4,000$ compared with the usual-care strategy (US\$8,127 vs. $\$ 12,145$ ). When including the US\$1,500 cost of the HeartFlow Analysis, total savings to the US healthcare system were projected to be $26 \%$ at 1 year. In the noninvasive testing stratum of
PLATFORM, the average costs of FFRcT were associated with slightly more use of invasive cardiac procedures and a trend towards higher medical costs, though it did not reach statistical difference (US\$2,679 vs. $\$ 2,137, \mathrm{P}=0.26$ ). Quality of life score, however, was more improved in FFR CT patients than in usual-care patients, which is probably because of more aggressive application of coronary revascularization for functionally significant coronary lesions detected by FFRct. These data suggest that introduction of FFRCT for the management of CAD patients has a potential benefit in Japan from the economic and patient care points of view.

\section{Clinical Utility of FFRct}

\section{Case 1: FFRcr as a Reliable Gatekeeper to CAG and Revascularization}

Figure 4 shows a case of a 70-year-old man with atypical chest pain at rest. Because cCTA showed intermediate stenosis of the mid-right coronary artery (RCA), the patient underwent CAG. CAG showed intermediate stenosis with a suggestive finding of plaque rupture in the mid-RCA without significant stenosis of the left coronary artery (LAD). In this case, FFRct was 0.92 and invasive FFR was 0.96, neither of which indicated the presence of myocardial ischemia. Therefore, if FFR $\mathrm{FT}_{\mathrm{C}}$ had been available before CAG, not only invasive FFR but also CAG would have been unnecessary for this patient.

\section{Case 2: Appropriate Decision-Making for PCI Without Invasive FFR}

A 77-year-old woman was admitted to Kobe University hospital because of effort angina. cCTA performed in the outpatient clinic showed intermediate stenosis with mild calcification (Figure 5). FFR ${ }_{\text {CT }}$ showed FFR $<0.80$ in the LAD without significant ischemia in the left circumflex (LCX) and RCA. The patient was sent to the catheterization laboratory and underwent CAG, which revealed an intermediate mid-LAD stenosis with invasive FFR of 0.72 
and she underwent revascularization by PCI on a subsequent day. If FFRct had been available before CAG, direct PCI immediately after CAG might have been possible. Knowing the presence of ischemia before angiography might reduce the radiation dose, procedure time, and enable the interventional cardiologist to concentrate on the PCI procedure.

\section{Case 3: Utility of FFRct With Diffuse Stenosis}

Currently, FFRct provides information on intracoronary pressure drop by using color-coded change from blue to red (Figure 6). This information is important for deciding the treatment strategy of CAD patients.

Figure 6 shows a case of diffuse coronary stenosis in RCA with significant LAD and LCX stenoses. Because the LAD and LCX lesions were relatively focal, simple stenting was selected. However, CAG showed diffuse intermediate narrowing without a significant focal stenosis in the entire RCA. FFRCT showed a gradual change of colorcoding from red to blue, suggesting a gradual pressure drop throughout the entire coronary artery. Therefore, optimal medical therapy was selected for the RCA lesion.

In most cases of diffuse intermediate stenosis, simple stenting to a specific lesion does not relieve the ischemia; hence bypass surgery or optimal medical therapy can be treatment options in such cases. In current clinical practice, we obtain the distribution of intracoronary pressure drop from the pressure-drop curve obtained by manual pull-back of a pressure wire. Although widely performed, this method has difficulties with registration between pressure changes and lesion location. On the other hand, $\mathrm{FFR}_{\mathrm{CT}}$ before CAG provides the information at a glance, which may help build and simplify treatment strategies for patients with CAD.

\section{Future Possibilities of FFRct: Interactive Planning Software and Total Plaque Force Analysis}

"Interactive planning" is a novel simulation of post-stent FFR to a specific coronary stenosis. With this technology, post-stent FFR can be predicted by re-calculation of hemodynamic parameters by eliminating ischemia-causing
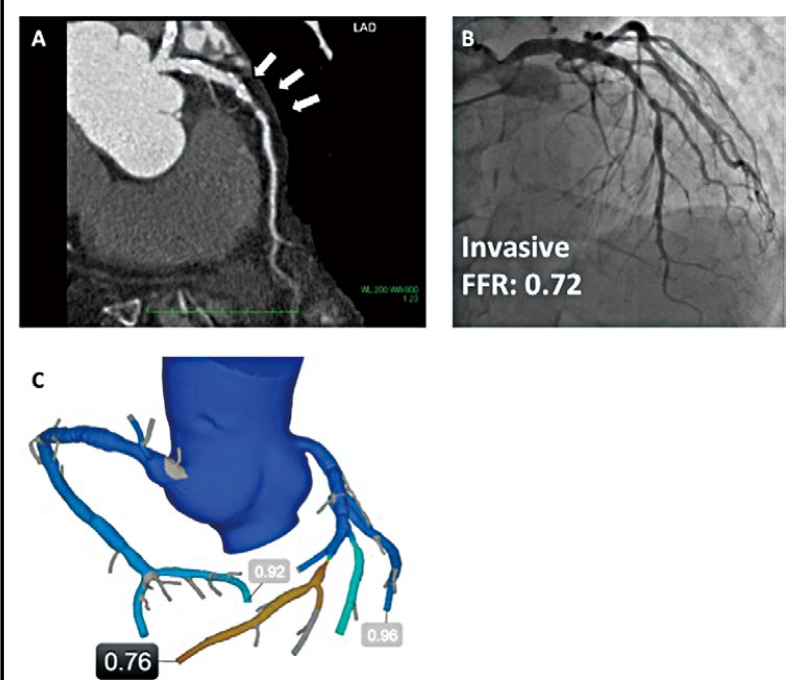

Figure 5. Case 2. (A) CTA image of $L A D$, (B) angiography of $\mathrm{LAD}$, and (C) FFRCT. Abbreviations as in Figures 1-3.
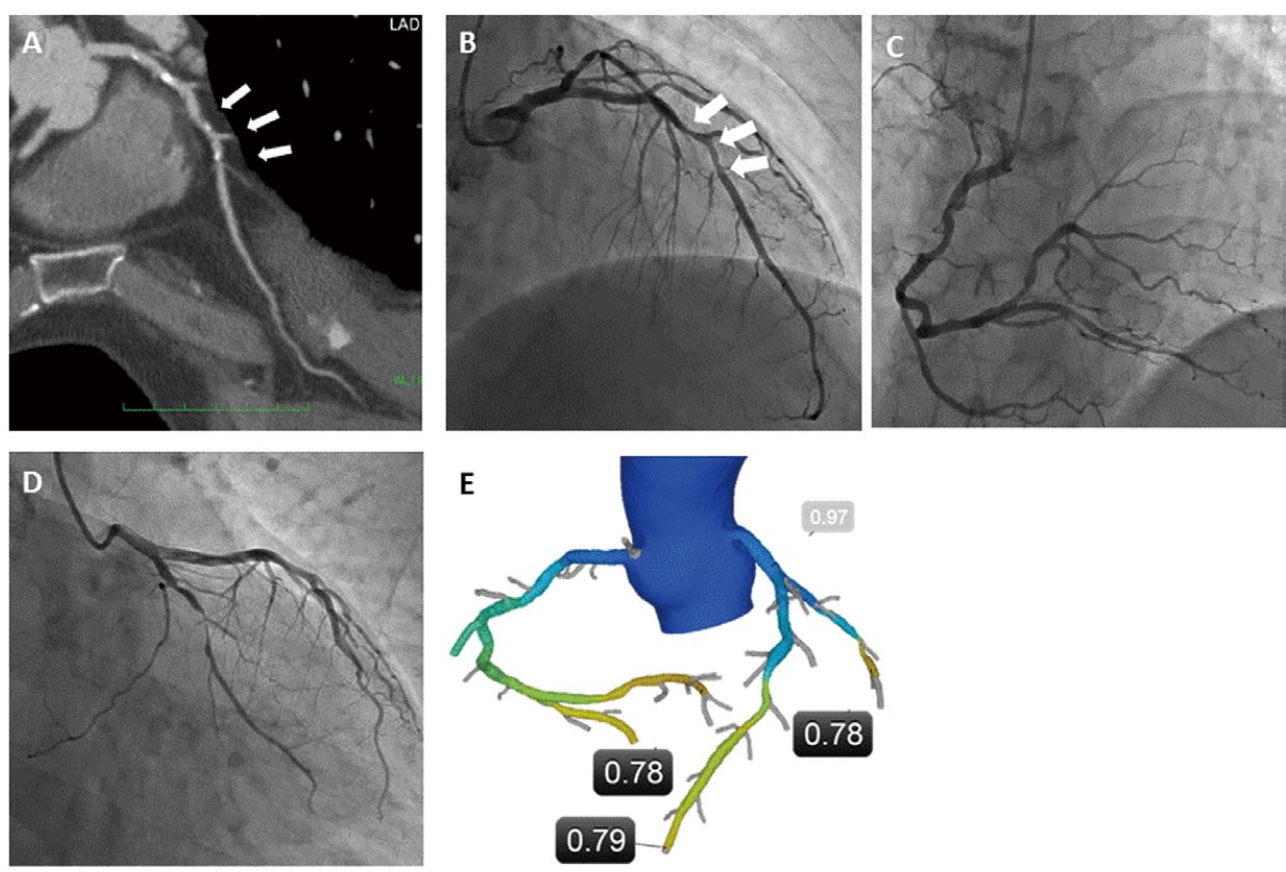

Figure 6. Case 3. (A) CTA image of LAD, (B) angiography of $L A D,(\mathbf{C}) R C A$, and (D) LCX, and (E) FFRCT. Abbreviations as in Figures 1-3. 
stenosis, serving to estimate the hemodynamic effect of stenting in a lesion-specific manner. Kim et al ${ }^{33}$ and $\mathrm{Koo}^{34}$ showed the feasibility and utility of this technology in predicting functional outcome after coronary stenting. The mean difference between FFR T $_{\text {and invasive FFR was }}$ 0.024 for post-stenting, and the diagnostic accuracy of FFR c $_{\text {to }}$ to predict ischemia after stenting was $96 \%$. Of note, post-stent FFR by interactive planning can be obtained using the original cCTA taken before the invasive procedure without any additional information needed. These simulations can be useful not only for decision-making (treat or not), but also for planning how to treat target lesions prior to PCI.

This novel technology can be expanded to improve understanding of rupture in association with the hemodynamic force acting on plaque and lesion geometry. In a recent clinical report, axial plaque stress (APS) was introduced by extracting the axial component of hemodynamic stress acting on stenotic lesions and evaluating the relationship with lesion morphology. ${ }^{35}$ In that study, upstream APS linearly increased as lesion severity increased, whereas downstream APS exhibited a concave function for lesion severity. These hemodynamic and geometric indices may be clinically helpful to assess the future risk of plaque rupture and to determine the treatment strategy for patients with CAD.

\section{Study Limitations}

Any diagnostic test has limitations. In the diagnostic accuracy trials performed for FFRc $\mathrm{F}_{\mathrm{C}}$ to date, exclusion criteria included recent myocardial infarction, prior stent implantation or coronary bypass surgery, contraindications to $\beta$-blockers, nitrates or adenosine, acute coronary syndrome, significant arrhythmia, and body mass index $>35 \mathrm{~kg} / \mathrm{m}^{2}$. Although patients with risk factors for microvascular disease (hypertension, diabetes) were included in the FFR clinical trials and these comorbidities were not significant predictors of accuracy in multivariate analyses, the accu-

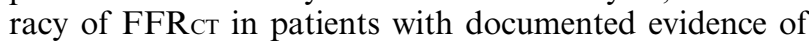
microvascular disease has not been studied. In such

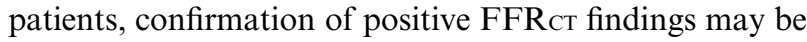
warranted by invasive FFR measurements in the cardiac catheterization laboratory.

Recently, Gaur et al reported the application of FFRCT to the evaluation of non-culprit vessels in patients with recent STEMI. ${ }^{36}$ They found that the diagnostic accuracy of FFR with suspected CAD and noted that the data did not support routine use of FFR $\mathrm{CT}$ in the post-STEMI setting.

\section{Conclusions}

FFRct enables calculation of lesion-specific ischemia without the need for an invasive procedure. Previous multicenter trials have consistently shown improved diagnostic accuracy over cCTA. Furthermore, in clinical studies and clinical practice, FFRст has been demonstrated to enable the safe deferral of up to $60 \%$ of diagnostic cardiac catheterization procedures. Considering that several clinical trials have shown its potential benefit in cost-effectiveness and symptom relief compared with usual care, introducing FFR $\mathrm{CT}$ to clinical practice might change the traditional approach to the management of patients with CAD. A large-scale clinical registry currently ongoing will provide further information on use of this technology in Japan.

\section{Conflict of Interest}

C.A.T. is an employee of and shareholder in HeartFlow, Inc., which provides FFRcT.

\section{References}

1. Tonino PA, Fearon WF, De Bruyne B, Oldroyd KG, Leesar MA, Ver Lee PN, et al. Angiographic versus functional severity of coronary artery stenoses in the FAME study fractional flow reserve versus angiography in multivessel evaluation. $\mathrm{J} \mathrm{Am} \mathrm{Coll}$ Cardiol 2010; 55: 2816-2821.

2. Fearon WF, Bornschein B, Tonino PA, Gothe RM, Bruyne BD, Pijls NH, et al. Economic evaluation of fractional flow reserveguided percutaneous coronary intervention in patients with multivessel disease. Circulation 2010; 122: 2545-2550.

3. De Bruyne B, Pijls NH, Kalesan B, Barbato E, Tonino PA, Piroth Z, et al. Fractional flow reserve-guided PCI versus medical therapy in stable coronary disease. N Engl J Med 2012; 367: 991-1001.

4. Kim HJ, Vignon-Clementel IE, Coogan JS, Figueroa CA, Jansen $\mathrm{KE}$, Taylor CA. Patient-specific modeling of blood flow and pressure in human coronary arteries. Ann Biomed Eng 2010; 38: 3195-3209.

5. Taylor CA, Figueroa CA. Patient-specific modeling of cardiovascular mechanics. Annu Rev Biomed Eng 2009; 11: 109-134.

6. Feldman RL, Pepine CJ, Conti CR. Magnitude of dilatation of large and small coronary arteries of nitroglycerin. Circulation 1981; 64: 324-333.

7. Abbara S, Arbab-Zadeh A, Callister TQ, Desai MY, Mamuya $\mathrm{W}$, Thomson L, et al. SCCT guidelines for performance of coronary computed tomographic angiography: A report of the Society of Cardiovascular Computed Tomography Guidelines Committee. J Cardiovasc Comput Tomogr 2009; 3: 190-204.

8. Johnson NP, Kirkeeide RL, Gould KL. Coronary anatomy to predict physiology: Fundamental limits. Circ Cardiovasc Imaging 2013; 6: 817-832.

9. Choi G, Uzu K, Toba T, Mori S, Takaya T, Shinke T, et al. TCT-333 Accuracy of lumen boundary extracted from coronary CTA for calcified and noncalcified plaques assessed using OCT data. J Am Coll Cardiol 2015; 66: B134.

10. Leipsic J, Yang TH, Thompson A, Koo BK, Mancini GB, Taylor C, et al. CT angiography (CTA) and diagnostic performance of noninvasive fractional flow reserve: Results from the Determination of Fractional Flow Reserve by Anatomic CTA (DeFACTO) study. Am J Roentgenol 2014; 202: 989-994.

11. Min JK, Taylor CA, Achenbach S, Koo BK, Leipsic J, Norgaard $\mathrm{BL}$, et al. Noninvasive fractional flow reserve derived from coronary CT angiography: Clinical data and scientific principles. JACC Cardiovasc Imaging 2015; 8: 1209-1222.

12. Taylor CA, Fonte TA, Min JK. Computational fluid dynamics applied to cardiac computed tomography for noninvasive quantification of fractional flow reserve: Scientific basis. J Am Coll Cardiol 2013; 61: 2233-2241.

13. Choy JS, Kassab GS. Scaling of myocardial mass to flow and morphometry of coronary arteries. J Appl Physiol 2008; 104: $1281-1286$

14. Gould KL, Johnson NP, Bateman TM, Beanlands RS, Bengel FM, Bober R, et al. Anatomic versus physiologic assessment of coronary artery disease: Role of coronary flow reserve, fractional flow reserve, and positron emission tomography imaging in revascularization decision-making. $J$ Am Coll Cardiol 2013; 62: $1639-1653$.

15. Kamiya A, Togawa T. Adaptive regulation of wall shear stress to flow change in the canine carotid artery. Am J Physiol 1980; 239: $\mathrm{H} 14-\mathrm{H} 21$.

16. Zarins CK, Zatina MA, Giddens DP, Ku DN, Glagov S. Shear stress regulation of artery lumen diameter in experimental atherogenesis. J Vasc Surg 1987; 5: 413-420.

17. Glagov S, Weisenberg E, Zarins CK, Stankunavicius R, Kolettis GJ. Compensatory enlargement of human atherosclerotic coronary arteries. $N$ Engl J Med 1987; 316: 1371-1375.

18. Wilson RF, Wyche K, Christensen BV, Zimmer S, Laxson DD. Effects of adenosine on human coronary arterial circulation. Circulation 1990; 82: 1595-1606.

19. Camici PG, Crea F. Coronary microvascular dysfunction. $N$ Engl J Med 2007; 356: 830-840.

20. De Bruyne B, Hersbach F, Pijls NH, Bartunek J, Bech JW, Heyndrickx GR, et al. Abnormal epicardial coronary resistance in patients with diffuse atherosclerosis but "normal" coronary 
angiography. Circulation 2001; 104: 2401-2406.

21. Sankaran S, Kim HJ, Choi G, Taylor CA. Uncertainty quantification in coronary blood flow simulations: Impact of geometry, boundary conditions and blood viscosity. J Biomech 2016; 49: $2540-2547$

22. Koo BK, Erglis A, Doh JH, Daniels DV, Jegere S, Kim HS, et al. Diagnosis of ischemia-causing coronary stenoses by noninvasive fractional flow reserve computed from coronary computed tomographic angiograms: Results from the prospective multicenter DISCOVER-FLOW (Diagnosis of Ischemia-Causing Stenoses Obtained Via Noninvasive Fractional Flow Reserve) study. J Am Coll Cardiol 2011; 58: 1989-1997.

23. Min JK, Leipsic J, Pencina MJ, Berman DS, Koo BK, van Mieghem C, et al. Diagnostic accuracy of fractional flow reserve from anatomic CT angiography. JAMA 2012; 308: 1237-1245.

24. Norgaard BL, Leipsic J, Gaur S, Seneviratne S, Ko BS, Ito H, et al. Diagnostic performance of noninvasive fractional flow reserve derived from coronary computed tomography angiography in suspected coronary artery disease: The NXT trial (Analysis of Coronary Blood Flow Using CT Angiography: Next Steps). $J$ Am Coll Cardiol 2014; 63: 1145-1155.

25. Miyoshi T, Osawa K, Ito H, Kanazawa S, Kimura T, Shiomi H, et al. Non-invasive computed fractional flow reserve from computed tomography (CT) for diagnosing coronary artery disease: Japanese results from NXT trial (Analysis of Coronary Blood Flow Using CT Angiography: Next Steps). Circ J 2015; 79: $406-412$.

26. Norgaard BL, Gaur S, Leipsic J, Ito H, Miyoshi T, Park SJ, et al. Influence of coronary calcification on the diagnostic performance of CT angiography derived FFR in coronary artery disease: A substudy of the NXT Trial. JACC Cardiovasc Imaging 2015; 8: $1045-1055$.

27. Gaur S, Bezerra HG, Lassen JF, Christiansen EH, Tanaka K, Jensen JM, et al. Fractional flow reserve derived from coronary CT angiography: Variation of repeated analyses. $J$ Cardiovasc Comput Tomogr 2014; 8: 307-314.

28. Douglas PS, De Bruyne B, Pontone G, Patel MR, Norgaard BL,
Byrne RA, et al. 1-year outcomes of FFRCT-guided care in patients with suspected coronary disease: The PLATFORM Study. J Am Coll Cardiol 2016; 68: 435-445.

29. Douglas PS, Pontone G, Hlatky MA, Patel MR, Norgaard BL, Byrne RA, et al. Clinical outcomes of fractional flow reserve by computed tomographic angiography-guided diagnostic strategies vs. usual care in patients with suspected coronary artery disease: The prospective longitudinal trial of FFR(CT): Outcome and resource impacts study. Eur Heart $J$ 2015; 36: 3359-3367.

30. Tanaka H, Chikamori T, Tanaka N, Hida S, Igarashi Y, Yamashita J, et al. Diagnostic performance of a novel cadmiumzinc-telluride gamma camera system assessed using fractional flow reserve. Circ J 2014; 78: 2727-2734.

31. Hlatky MA, Saxena A, Koo BK, Erglis A, Zarins CK, Min JK. Projected costs and consequences of computed tomographydetermined fractional flow reserve. Clin Cardiol 2013; 36: $743-748$.

32. Kimura T, Shiomi H, Kuribayashi S, Isshiki T, Kanazawa S, Ito $\mathrm{H}$, et al. Cost analysis of non-invasive fractional flow reserve derived from coronary computed tomographic angiography in Japan. Cardiovasc Interv Ther 2015; 30: 38 -44.

33. Kim KH, Doh JH, Koo BK, Min JK, Erglis A, Yang HM, et al. A novel noninvasive technology for treatment planning using virtual coronary stenting and computed tomography-derived computed fractional flow reserve. JACC Cardiovasc Interv 2014; 7: $72-78$.

34. Koo BK. The present and future of fractional flow reserve. Circ J 2014; 78: $1048-1054$

35. Choi G, Lee JM, Kim HJ, Park JB, Sankaran S, Otake H, et al Coronary artery axial plaque stress and its relationship with lesion geometry: Application of computational fluid dynamics to coronary CT angiography. JACC Cardiovasc Imaging 2015; 8: $1156-1166$.

36. Gaur S, Taylor CA, Jensen JM, Bøtker HE, Christiansen EH, Kaltoft AK, et al. FFR derived from coronary CT angiography in nonculprit lesions of patients with recent STEMI. JACC Cardiovasc Imaging 2017; 10: 424-433. 